\title{
Influencia del tamaño de los carburos del acero para cojinetes de bolas relacionada con estabilización de diámetros y las propiedades mecánicas después del tratamiento térmico final
}

\author{
J.W. Filipek* y E. Rincón*
}

\begin{abstract}
Resumen El presente trabajo esta dando un enfoque específico de lo que es la influencia de la estructura de la materia prima (dispersión y forma de los carburos) en estado recocido para la cantidad de austenita retenida, la dureza y otras propiedades después del tratamiento térmico final (temple y revenido). Se observó, con base en los resultados, que existe una estrecha relación entre las estructuras (tamaño y forma de carburos) y las propiedades mecánicas.
\end{abstract}

Palabras clave Microestructura. Cementita. Austenita. Carburos. Temple y revenido.

Influence of the size of the annealing carbons in steel for ball bearings in relation with stabilisation of diameters and mechanical properties after the final thermic treatment

\begin{abstract}
A high quality steel for rollez production is a function of several properties including its mechanical properties, cleanness, structure as well as thermal and mechanical treatments. Accordingly, this paper presents a study of the influence of the material microestructure of the annealing state on the effectiveness of the thermal treatment for quenching and tempering and the final properties.
\end{abstract}

Keywords Microstructure. Cementite. Austenite. Carbides. Composition. Steel.

\section{INTRODUCCION}

El acero es lo más importante de las aleaciones metálicas por sus buenas propiedades como son su gran plasticidad y maleabilidad a elevadas temperaturas, alta resistencia al desgaste, etc. Este material, a través del tratamiento térmico, puede lograr también excelente maquinabilidad, así como buenas propiedades mecánicas, las cuales se caracterizan por alta dureza, alta resistencia y tensidad, dando finalmente una vida muy prolongada de los elementos de construcción en función del tiempo de trabajo. En esta investigación se analizó la influencia de diferentes estructuras obtenidas en el proceso de recocido para la vida útil de los elementos finales (rodamientos). Se observó, con base en los resultados, que existe una estrecha relación entre las estructuras (tamaño de carburos) en estado recocido y las propiedades mecánicas de elementos finales después de temple y revenido así como, también, la estabilización de diámetros de los elementos tratados térmicamente.

\section{MATERIALES Y PROCEDIMIENTO EXPERIMEN- TAL}

En esta investigación se analizó el acero grado construcción tipo 52100 para rodamientos según la norma ASTM-A535- 79 cuya composición química presenta la tabla siguiente.

Tabla I. La composición química de acero, de acuerdo a la norma ASTM

Table I. Chemical Composition of steel according to ASTM norm

\begin{tabular}{lcccccccc}
\hline $\begin{array}{l}\text { Colada } \\
\mathrm{n}^{\circ} 13028 \\
\%\end{array}$ & $\mathbf{C}$ & $\mathbf{M n}$ & $\mathbf{S i}$ & $\mathbf{C r}$ & $\mathbf{P}$ & $\mathbf{S}$ & $\mathbf{N i}$ & $\mathbf{C u}$ \\
\cline { 2 - 8 } & 1,1 & 0,3 & 0,31 & 1,34 & 0,017 & 0,015 & 0,16 & 0,1 \\
\hline
\end{tabular}

(*) Facultad de Ingeniería ,Universidad Autónoma del Estado de México, Cerro de Coatepec S/N , 50130 , Toluca Estado de México. Tel: (722) 2140855, Email: jwf@uaemex.mx 
Para el análisis del problema se preparó una colada del acero 52100. Después de la laminación, el material fue dividido en 4 partes con propósito de preparar distintas estructuras de diferente forma y dispersión de los carburos en estado recocido. Las microestructuras en estado recocido, así como en estado templado y revenido pueden ser observadas en el anexo.

\section{RESULTADOS}

Con base en los resultados obtenidos se puede decir que la forma y dispersión de los carburos en estado recocido tiene gran influencia para los parámetros dureza, maquinabilidad, tamaño del grano austenítico, composición química de la austenita y, finalmente, es uno de los principales factores para la cinética del proceso de austenitización. Presentando la influencia de diferentes microestructuras del mismo acero para resultados finales, después del temple y revenido, se debe mencionar que el acero tipo 2100 en estado recocido tiene de 15 a $16 \%$ de los carburos, los cuales contienen $9 \% \mathrm{Cr}$, mientras la matriz ferrítica contiene, solamente, 0,2 \% Cr y $0,03 \%$ C. Después del temple desde la temperatura de $850^{\circ} \mathrm{C}$ durante $30 \mathrm{~min}$. de austenización, la cantidad de los carburos tienen un valor aproximado de $8,5 \%$, lo que se presenta en la tabla II.

También, se hizo la medición de la dureza del acero en los cuatro grupos, tanto en estado recocido como templado y revenido, cuyos resultados se presentan en la tabla III.

Con base en las pruebas del acero 52100 con diferentes estructuras en estado recocido, templadas desde $850^{\circ} \mathrm{C}$ y revenidas en $150^{\circ} \mathrm{C}$ durante, $2 \mathrm{~h}$, se obtuvieron las siguientes cantidades de austenita residual (Tabla IV).

Tabla II. Cantidad de los carburos en estado recocido y templado, dependiendo del grado de dispersión

Table II. Quantity of carbides in annealing and quenching state depending on the degree of dispersion

\begin{tabular}{lcc}
\hline No. de Probeta & \multicolumn{2}{c}{ Vp 100 (\%) de volumen } \\
\cline { 2 - 3 } & $\begin{array}{c}\text { \% de volumen } \\
\text { de los carburos } \\
\text { en estado recocido }\end{array}$ & $\begin{array}{c}\text { \% de volumen } \\
\text { de los carburos } \\
\text { en estado templado }\end{array}$ \\
\hline 3036 & 16,034 & 8,6 \\
3037 & 16,4 & 8,7 \\
3038 & 16,2 & 8,22 \\
3039 & 16,48 & 8,4 \\
\hline
\end{tabular}

Tabla III. Dureza HB del material en estado recocido y estado templado HRC, Colada N 13028

Table III. HB Hardness of material in annealing and HRC quenching state

\begin{tabular}{llll}
\hline $\begin{array}{l}\text { Estado del } \\
\text { material }\end{array}$ & $\begin{array}{c}\text { Grupo de los } \\
\text { materiales }\end{array}$ & \multicolumn{2}{c}{$\begin{array}{c}\text { Dureza de los grupos } \\
\text { en estado: }\end{array}$} \\
\cline { 3 - 4 } & & Recocido & Templado \\
\hline Recocido & $3036-\mathrm{RI}$ & HBmin-197 & HBmax-201 \\
& $3037-\mathrm{RII}$ & HBmin-187 & HBmax-197 \\
& $3038-\mathrm{RIII}$ & HBmin-174 & HBmax-183 \\
& $3039-\mathrm{RI}$ & HBmin-174 & HBmax-197 \\
\hline Templado y & & & \\
revenido & $3036-\mathrm{HI}$ & HRCmin-61 & HRCmax-62 \\
& $3037-\mathrm{HII}$ & HRCmin-61 & HRCmax-61,5 \\
& $3038-\mathrm{HIII}$ & HRCmin-60,5 & HRCmax-61,5 \\
& $3039-\mathrm{HIV}$ & HRCmin-62 & HRCmax-63 \\
\hline
\end{tabular}

1 = Estructura de cementita globular muy fina

$\|=$ Estructura de cementita globular fina

III = Estructura de cementita globular gruesa

IV = Estructura de cementita globular con la perlita

Tabla IV. Cantidad de austenita residual, dependiendo de la microestructura inicial

Table IV. Quantity of the residual austenite depending on the initial microestructure

\begin{tabular}{lc}
\hline Tipo de la estructura & $\begin{array}{c}\% \text { de cantidad } \\
\text { de la austenita } \\
\text { residual }\end{array}$ \\
\hline a) Estructura de cementita globular fina & $14 \%$ \\
b) Estructura de cementita globular gruesa & $8 \%$ \\
c) Estructura de cementita globular y perlita & $18 \%$ \\
\hline
\end{tabular}

Como se ve, con base en los datos, la estructura de la materia prima afecta para la cantidad de austenita retenida, después de temple y revenido. La cantidad de la austenita retenida según Spector ${ }^{[2]}$, la cual garantiza estabilidad de diámetros a las temperaturas de trabajo bajo cero, después del temple y revenido, se presenta en la tabla V.

La forma ilustrativa de la influencia de la austenita residual a la temperatura ambiente para estabilidad de diámetros, se presenta en la figura 1. 
Influencia del tamaño de los carburos del acero para cojinetes de bolas relacionada con estabilización de diámetros y las propiedades mecánicas después del tratamiento térmico final

J.W. FILIPEK Y E. RINCÓN

Tabla V. Cantidad de austenita residual con cambios aceptados de diámetros, $\left(1 \times 10^{-5} \mathrm{~mm} / \mathrm{mm}\right)$ Revenido a $150^{\circ} \mathrm{C}, 2 \mathrm{~h}$ Table V. Quantity of residual austenite with the accepted diameter changes $\left(I \times 10^{-5} \mathrm{~mm} / \mathrm{mm}\right)$

\begin{tabular}{lcccc}
\hline Material & Temperatura del temple en aceite ${ }^{\circ} \mathrm{C}$ & \multicolumn{2}{c}{$\%$ de austenita residual estable } \\
\cline { 3 - 5 } & & $-50{ }^{\circ} \mathrm{C}$ & $\mathbf{- 2 0 0}{ }^{\circ} \mathrm{C}$ & Temp. ambiente durante 5 años \\
\hline Acero 52100 & 840 & 14 & 10 & 10 \\
& 860 & 16 & 14 & 15 \\
\hline
\end{tabular}

\section{$1 \mathrm{~m} / 100 \mathrm{~mm}$}
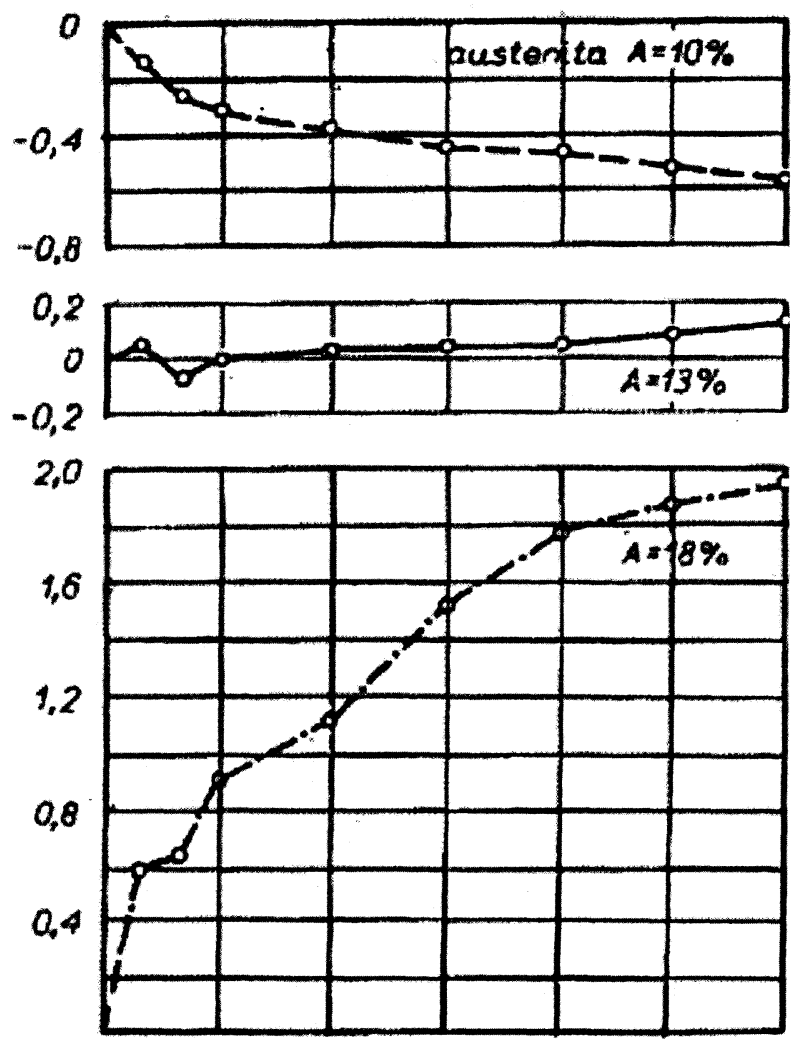

Figura 1. Cambio de diámetros de las probetas del acero 52100 , con diferente cantidad de austenita residual. Las probetas después de temple y revenido, fueron estabilizadas en la temperatura de $120^{\circ} \mathrm{C}$, durante $90 \mathrm{~h}$.

Figure 1. Change of the diameter of steel simples 52100, with different quantities of residual austenite. The simples after the operations of tempering and quenching were stabilized at the temperature of $120^{\circ} \mathrm{C}$ during $90 \mathrm{~h}$.

\section{DISCUSIÓN}

\section{T. Influencia del grado de dispersión de los carburos en estado recocido, para la canti- dad de la austenita residual y cambio de diámetros}

La estructura y forma de los carburos en estado recocido tiene influencia principal para la homogeneidad de la austenita, proceso de templabilidad, cantidad de austenita residual después del tratamiento térmico final y estabilidad de diámetros. La figura 2 presenta la influencia de la microestructura en estado recocido del acero 52100 para:

- la cantidad de austenita residual

- tamaño del grano de la austenita

- dureza después del temple y revenido en 150 ${ }^{\circ} \mathrm{C}$, durante $2 \mathrm{~h}$

Con base en el diagrama presentado se puede decir que, la estructura de cementita fina en estado recocido da grano fino de austenita, a temperaturas entre $840-850^{\circ} \mathrm{C}$. Con aumento de la temperatura de austenitización, las estructuras laminares (de la perlita) presentan mayor grado de crecimiento de grano austenítico, comparando con estructuras globulares. Esto, finalmente, afecta a las propiedades mecánicas, especialmente de la fatiga ${ }^{[2]}$. El grano más fino está presente en el grupo de probetas con estructura globular fina.

Considerando el análisis sobre la influencia de dispersión y forma de los carburos en estado recocido, se debe enfocar que las estructuras con alto grado de dispersión, aunque aumentan la cantidad de austenita residual, nos dan la posibilidad de obtener elementos con dureza por encima de $60 \mathrm{HRC}$, así como en grano muy fino. Estos parámetros garantizan la gran resistencia a la fatiga y la disminución de las fracturas tipo frágil. La estructura de la materia prima tiene también gran influencia para el tamaño del grano y se presenta en la tabla VI.

Para analizar la duración de las probetas que tenían formas cónicas, primero, fueron templadas y revenidas con la siguiente tecnología:

- Calentamiento TI hasta $200{ }^{\circ} \mathrm{C}$, tiempo: 15 $\min$

- Calentamiento T2 hasta $680{ }^{\circ} \mathrm{C}$, tiempo: 12 $\min$

- Calentamiento T3 hasta $850{ }^{\circ} \mathrm{C}$, tiempo: 18 $\min$ 

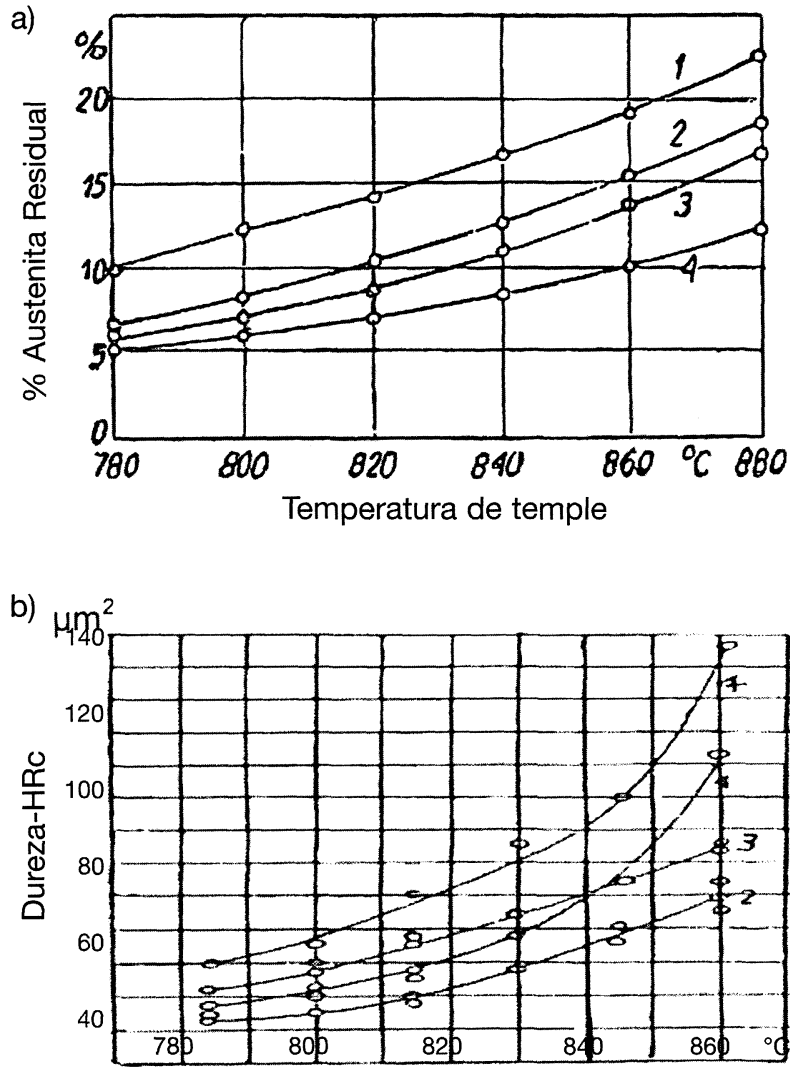

Temperatura de temple

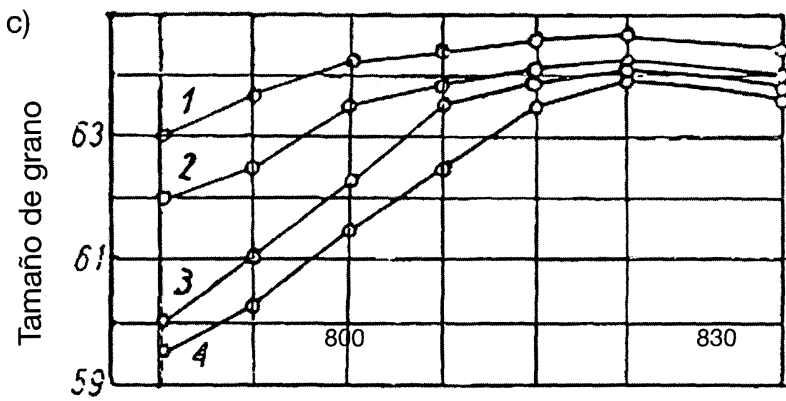

Temperatura de temple

Figura 2. Influencia de la microestructura del acero en estado recocido y las diferentes temperaturas de austenitización (tiempo de austenitización $15 \mathrm{~min}$ ), sobre: a) contenido de austenita residual, b) tamaño del grano austenítico, c) dureza después del temple y revenido (revenido $150^{\circ} \mathrm{C}$, 2h).

Figure 2. Influence of the microstructure of steel 52100 at annealing state and temperature of austenization (time of austenization $15 \mathrm{~min}$ ) on: a) quantity of residual austenite, b) size of austenitic grain , c) hardness after tempering and (quenching at $150^{\circ} \mathrm{C}, 2 \mathrm{~h}$ ).

- Enfriamiento T4 $40-70^{\circ} \mathrm{C}$, tiempo: $15 \mathrm{~min}$

- Revenido T5 hasta $160^{\circ} \mathrm{C}$, tiempo: $180 \mathrm{~min}$

- Revenido bajo T6 hasta $140{ }^{\circ} \mathrm{C}$, tiempo: 360 $\min$
Tabla VI. Tamaño de grano de austenita en función de la forma de la cementita

Table VI. Size of Austenite en function of the form of the cementite

\begin{tabular}{|c|c|c|c|}
\hline $\begin{array}{l}\text { Estado } \\
\text { material }\end{array}$ & $\begin{array}{c}\text { No de probetas } \\
\text { según } \\
\text { estructuras }\end{array}$ & $\begin{array}{l}\text { Tamaño del } \\
\text { grano de } \\
\text { austenita } \\
\text { ASTM }\end{array}$ & $\begin{array}{l}\text { Promedio del } \\
\text { diámetro de } \\
\text { grano austenítico } \\
(\mathrm{mm})\end{array}$ \\
\hline & 3036-HII & & \\
\hline & $\begin{array}{c}\text { cementita muy fina } \\
3037-\mathrm{HII}\end{array}$ & $9 / 8$ & 0,017 \\
\hline $\begin{array}{c}\text { Templado } \\
\text { y }\end{array}$ & $\begin{array}{c}\text { cementita media } \\
\text { 3038-HII }\end{array}$ & $7 / 8$ & 0,0265 \\
\hline \multirow[t]{2}{*}{ Revenido } & $\begin{array}{c}\text { cementita gruesa } \\
\text { 3039-HIV }\end{array}$ & 7 & 0,031 \\
\hline & $\begin{array}{l}\text { cementita con la } \\
\text { perlita }\end{array}$ & $6 / 8$ & 0,039 \\
\hline
\end{tabular}

Después del tratamiento térmico y rectificado, las probetas se investigaron en el aparato SetaShell. El movimiento de fatiga se obtiene en la primera ruptura, este momento se registra por cambio del sonido de trabajo de este equipo.

$$
F_{(t)}=1-e^{-(t / T)^{k}}
$$

donde: $F_{(t)}=$ Probabilidad de que la probeta muestre su fatiga en el tiempo $t$

$\mathrm{K}=$ Parámetro que caracteriza los valores del material

$\mathrm{T}=$ Parámetro que caracteriza los valores de construcción

$\mathrm{t}=$ Tiempo

Con base en los valores de duración $\mathrm{L}_{10}$ y $\mathrm{L}_{50}$, obtenidos como resultado de la investigación utilizando la fórmula de Weibull, se obtuvieron las líneas que muestran la duración de las probetas y la influencia del grado de dispersión de los carburos sobre la duración de los cojinetes. Estos resultados, preparados estadísticamente, se presentan en el correspondiente diagrama (Fig. 3) y en la tabla VII.

\section{CONCLUSIONES}

Como resultado de esta investigación se puede decir lo siguiente:

- Existe una estrecha relación entre el grado de dispersión de los carburos en estado recocido y la dispersión de la estructura martensítica. 
Influencia del tamaño de los carburos del acero para cojinetes de bolas relacionada con estabilización de diámetros y las propiedades mecánicas después del tratamiento térmico final

J.W. FILIPEK Y E. RINCÓN

Tabla VII. La influencia del grado de dispersión de los carburos en la duración del trabajo de los cojinetes

Table VII. The influence oft he degree of the dispersion of carbides in the time of pitting of bearing

\begin{tabular}{cccccc}
\hline Estado del Material & Grupo del Material & $\begin{array}{c}\text { Diámetro medio } \\
\text { de los Carburos } \\
(\mathbf{m m})\end{array}$ & $\begin{array}{c}\text { Duración convencional } \\
\mathrm{L}_{10} \text { Min / \% }\end{array}$ & $\begin{array}{c}\text { Duración media } \\
\mathrm{L}_{50} \text { Min / \% }\end{array}$ & $\begin{array}{c}\text { Diámetro medio } \\
\text { grano Austentita } \\
\text { (mm) }\end{array}$ \\
\hline Templado & $\mathrm{H} 1$ & $11 \times 10^{-4}$ & $117,13 / 393$ & $232,64 / 325,7$ & 0,017 \\
y & $\mathrm{H} 2$ & $14,5 \times 10^{-4}$ & $79,86 / 267$ & $165,63 / 241,2$ & 0,0265 \\
Revenido & $\mathrm{H} 3$ & $16,2 \times 10^{-4}$ & $29,87 / 100 \%$ & $68,67 / 100$ & 0,031 \\
& $\mathrm{H} 4$ & No se preparo & $23,55 / 87,50$ & $91,58 / 133,4$ & 0,039 \\
\hline
\end{tabular}

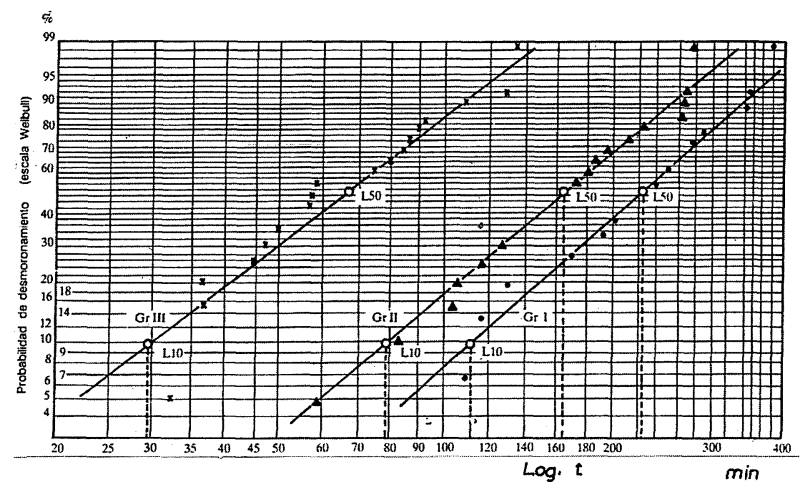

Figura 3. Diagrama del desmoronamiento en función del tiempo de trabajo.

Figure 3. Diagram of the pitting as a function of the working time.

- Los materiales con carburos muy finos tienen alto grado de saturación con carbono y cromo, lo que finalmente aumenta propiedades mecánicas.

- La estructura de materia prima con carburos finos presenta mejor estabilización de diámetros en función del tiempo, tanto en temperaturas bajas como en medio ambiente.

- Los materiales que tienen alto grado de dispersión de los carburos en estado revenido dan, después de temple y revenido, grano más fino, aumentando las propiedades de la fatiga hasta cuatro veces ${ }^{[I]}$.

\section{ANEXO}

Las siguientes micrografías corresponden a las microestructuras en estado recocido (Fig. 4) y en estado templado y revenido (Fig. 5).

\section{REFERENCIAS}

[1] J. WÓJIK, $14^{\text {th }}$ Rolling Conf. IAS, San Nicolás Argentina, 2002, pp 229-238.
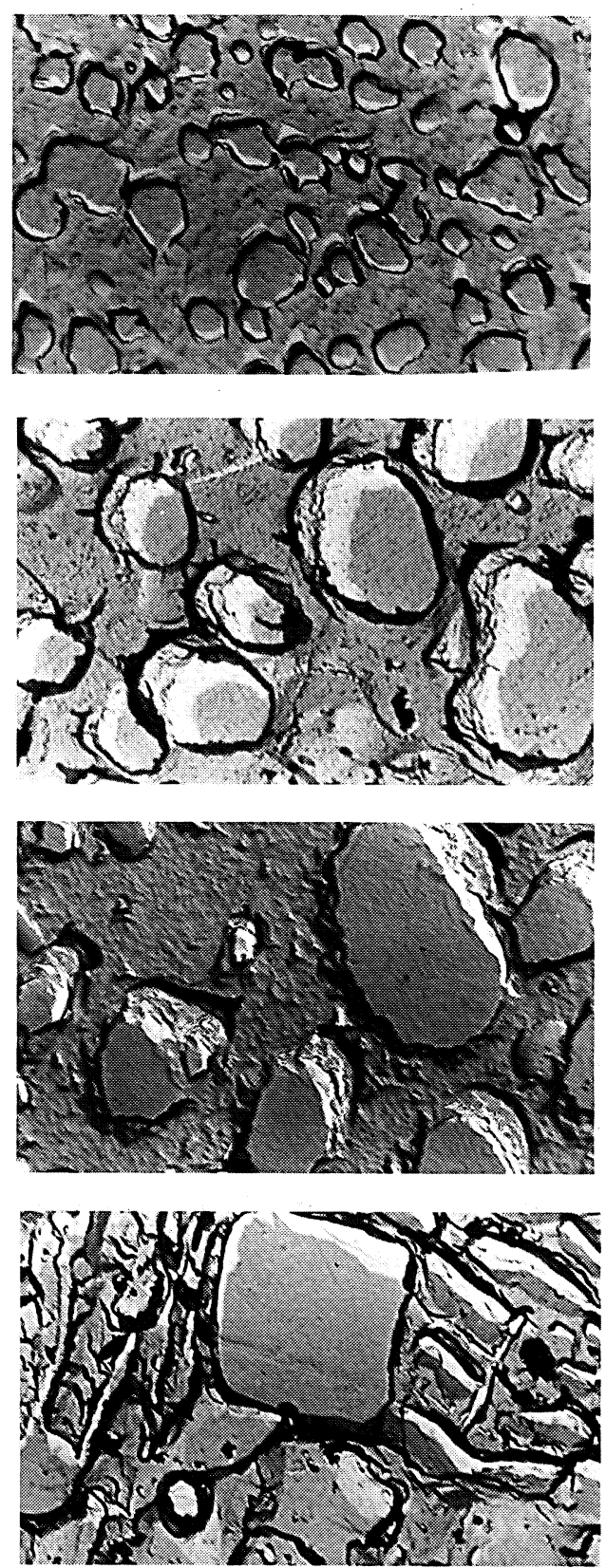

Figura 4. Las Microestructuras en estado Recocido $R$ (con un aumento de 5.000 x-microscopio electrónico).

Figure 4. The microstructure in the annealing state (increase 5000x).

Rev. Metal. Madrid Vol. Extr. (2005) 324-329 

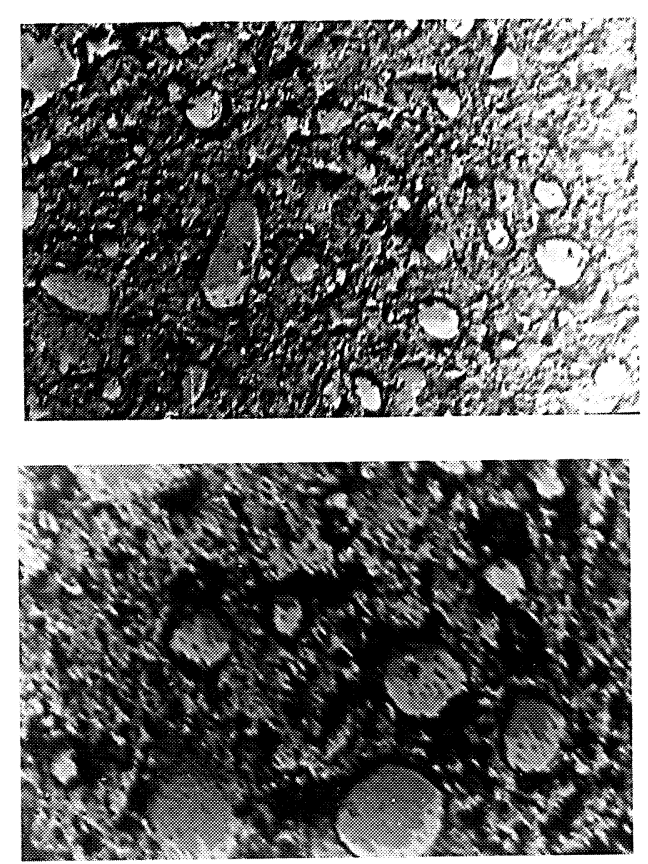
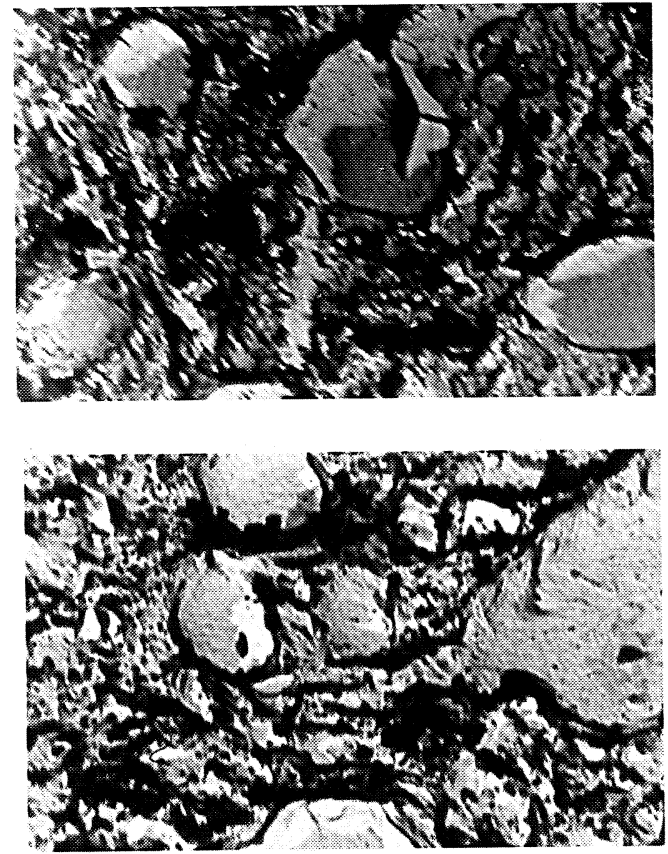

Figura 5. Las microestructuras en estado templado y revenido T (con un aumento de 5,000 x-microscopio electrónico).

Figure 5. The microstructure in the tempering and guenching state (increase 5000x).

[2] W. LuTY, Stale Lozyskowe, Ed. W.N.T. Warszawa, 1996.

[3] S. ButNickI, Spawalnosc, Kruchosc Stali, Ed. W.N.T. Warszawa, 1991.
[4] Y.M. Lajtin, Metalografía y Tratamiento Térmico de los Metales, Ed. Mir, 1985.

[5] L.A. DobrZANSKI, Metaloznawstwo z Podstawami nuuki o materialach, Ed. N.T. Warszawa, 1996. 